\title{
CONTACTS DE LANGUES ET GEOPOLITIQUE DES LANGUES ROMANES
}

\author{
Louis-Jean Calvet
}

\begin{abstract}
RÉSUMÉ
Dans cet article nous concevons premièremenet le contact entre les langues à partir de différentes configurations pour, ensuite, analyser la géopolitique des langues romanes, représentées par les trois grands ensembles linguistiques, soit, les ensembles francophone, hispanophone et lusophone.
\end{abstract}

MOTS-CLÉS: langues en contact; langues romanes; politique linguistique

i les contacts entre langues sont aussi vieux que les langues elles-mêmes, l'approche scientifique de la notion de langues en contact est plus récente : elle semble remonter à Uriel Weinreich et à son Languages in contact ${ }^{1}$. Il y traitait essentiellement des effets sur les langues de leur coexistence, en particulier des interférence. Mais lorsqu'ils parlaient de bilinguisme, Weinreich et son préfacier André Martinet entendaient essentiellement le bilinguisme individuel, et Weinreich, s'intéressant essentiellement aux interférences chez des individus bilingues, étudiait les effets du bilinguisme sur la forme des langues. Ce faisant, sans le savoir bien sûr, il mettait l'accent sur les limites d'une linguistique uniquement interne.

Quelques années plus tard, en 1956, Marcel Cohen dans son Pour une sociologie du langage ${ }^{2}$ proposait une approche beaucoup plus large et en 1959 Charles Ferguson présentait son concept de Diglossia ${ }^{3}$ qui donnait l'image

\footnotetext{
U. Weinreich, Languages in Contact : Findings and Problems, Mouton, La Haye, 1953

2 Paris, Albin Michel, 1956

3 "Diglossia", Word, 15, 1959
} 
d'une coexistence harmonieuse entre deux langues ou deux formes d'une langue, d'une répartition fonctionnelle des usages, chacune des langues ou des formes ayant ses fonctions. Cette idée de "coexistence harmonieuse" sera très vite critiquée en Europe, en particulier par les linguistes catalans et occitans, arguant que les contacts ou les rapports entre langues n'étaient pas harmonieux mais conflictuels. Et Ferguson lui-même fera d'ailleurs une sorte d'autocritique en revisitant de façon critique sa notion de diglossie ${ }^{4}$.

\section{Diverses configurations de la situation linguistique du monde}

Mais il y a d'autres façons de concevoir ces contacts. On se trouve en effet, quand on tente de présenter la situation linguistique du monde, face à différentes possibilités. La première est une configuration gravitationnelle , qui fait apparaître une langue hypercentrale, aujourd'hui l'anglais, autour de laquelle gravitent des langues supercentrales, à leur tour pivot de gravitation des langues centrales autour desquelles gravitent des milliers de langues périphérique. On peut aussi penser à une configuration génétique. C’est un lieu commun de dire qu'il existe entre les langues du monde un certain nombre de ressemblances ou de différences et les linguistes parlent à ce propos de "familles linguistiques”. Ces parentés génétiques peuvent favoriser des rapprochements, dans le cadre d'intégrations régionales par exemple. L'espagnol ou l'italien sont plus «transparents» pour un francophone que l'allemand ou le suédois, et cette transparence facilite à la fois la communication interpersonnelle et les relations entre États. On peut ici songer aux rapports entre l'espagnol et le portugais dans le cadre du MERCOSUL : l'intercompréhension relativement aisée entre ces deux langues et la volonté d'enseigner l'espagnol au Brésil et le portugais dans les pays hispanophones peuvent aider de façon évidente à l'intégration. On pourrait également imaginer dans le cadre de la Communauté européenne une politique linguistique "latine" entre l'Espagne, la France, l'Italie et le Portugal: la situation de communication inter-frontalière peut être conservée, voire améliorée, lorsque les langues concernées sont apparentées. Mais cet apparentement ne suffit pas toujours. Il y a par exemple entre le Danemark, la Norvège et la Suède une communication inter-scandinave potentielle, due à la

\footnotetext{
4 "Diglossia revisited", Southwest Journal of Linguistics 10, 1991.

5 Voir Louis-Jean Calvet, Pour une écologie des langues du monde, Patis, Plon, 1999, p. 75-98.
} 
relative unité linguistique, communication que les gouvernements prétendent favoriser mais que l'engouement des populations pour l'anglais semble mettre à mal. Comme l'écrit un spécialiste des langues scandinaves:

S'il existe bien, en effet, au niveau des gouvernements une volonté de convergence linguistique et des agences officielles (...) chargées de lui donner corps, ainsi qu'un enseignement scolaire des "langues voisines" dans chaque pays, il faut bien reconnaître que le taux d'adhésion populaire à la cause en question est plutôt faible et, malheureusement, inversement proportionnel à l'enthousiasme démesuré que l'on arbore, chez les jeunes en particulier, pour l'anglo-américain et la «culture» qu'il véhicule $^{6}$

La langue de la mondialisation vient ainsi s'immiscer dans une communication potentielle, comme un grain de sable venant gripper des rouages: le marché aux langues est donc ici truqué et le choix forcé. En revanche la configuration politique nous révèle de possibles foyers de résistance face à ces effets de la mondialisation. Il y a dans le monde au moins cinq grands ensembles linguistiques, cinq groupes de pays réunis par une même langue officielle: l'arabe, le français, l'espagnol, l'anglais et le portugais. Certains de ces ensemble sont organisés (c'est le cas de la Francophonie...) d'autres le sont moins, mais ils n'en constituent pas moins des réalités que j'ai proposé d'appeler «Xphonies» (sur le modèle de Francophonie: Arabophonie, Hispanophonie, etc...). Or les parentés génétiques et ces Xphonies tissent dans la configuration gravitationnelle d'autres liens que ceux qui procèdent des rapports de force et du seul système des bilinguismes. Le tableau des rapports entre les langues que nous propose la configuration gravitationnelle pourrait en effet paraître figé, mais ces rapports sont traversés par d'autres forces, d'autres liens. Les parentés génétiques tissent ainsi des rapports transversaux entre certaines langues (les langues romanes ou scandinaves, bien sûr, mais aussi les arabes et l'hébreu, le hindi et l'ourdou, les langues mandingues, les langues bantoues, etc...). Le mbochi par exemple, langue bantoue parlée au Congo, est une langue

6 Chritophe Bord, Langues scandinaves, Paris, PUF, 2001, page 114 
périphérique qui gravite autour du lingala, langue centrale qui elle-même gravite autour du français qui gravite autour de l'anglais. Mais, en mêmetemps, le mbochi est génétiquement lié à d'autres langues périphériques de la famille bantoue, comme le kirundi par exemple, ainsi qu'à des langues centrales à fonction véhiculaire comme le lingala, le munukutuba, le kiswahili, etc..., et cet ensemble constitue une réalité sur laquelle pourraient jouer des politiques linguistiques régionales.

Nous avons donc un certain nombre de configurations reposant sur des principes organisateurs différents: une configuration gravitationnelle (reposant sur les systèmes de bilinguisme), une configuration génétique (reposant sur la parenté génétique entre les langues), une configuration politique (reposant sur les langues utilisées pour la gestion des États). Et il en est d'autres possibles: nous pourrions par exemple configurer la situation linguistique mondiale du point de vue des systèmes graphiques utilisés (alphabets latin, cyrillique, devanagari, arabe, caractères chinois, etc.), du point de vue des lois linguistiques (absence ou présence de lois, typologie de ces lois, etc.), ou du point de vue véhiculaire (langues véhiculaires nationales ou transfrontalières), etc.

Dans chacun de ces cas, il s'agit d'une opération descriptive et heuristique qui permet de représenter une situation d'un certain point de vue, sous un certain angle, sans en modifier les constituants mais en les organisant d'une certaine façon. Ainsi, en classant dans mon ordinateur les éléments d'un dossier par ordre chronologique, j'ai une certaine vision de mon travail intellectuel récent, et j'en ai une autre vision en les classant par taille: le même document va prendre selon les cas un sens dans la progression de mon travail (c'est par exemple par lui que j'ai commencé) ou un autre sens dans l'importance accordée aux différents thèmes ( le document a une taille de 50, 100 ou 500 Ko par exemple). Une configuration ne consiste donc pas seulement à prendre acte d'un état de fait, elle est une intervention transitive sur les faits, une présentation parmi d'autres possibles, en fonction d'une logique qui donne à ces faits une certaine forme, un certain sens. Ainsi, en prenant comme principe organisateur la parenté génétique romane nous obtenons une configuration du globe nous donnant à voir par exemple que l'Amérique latine ne s'étend pas seulement du Chili au Mexique mais qu'elle englobe une partie des Caraïbes (Martinique, Guadeloupe, Haïti, etc.) et du Canada (Québec, Nouveau Brunswick, etc.). De la même façon une configuration des langues du monde en fonction des systèmes graphiques nous 
révélerait des zones de conflits potentiels ou effectifs: en Russie l'enclave latine du Tatarstan dans un environnement cyrillique, ou en Inde l'opposition alphabet arabe/alphabet devanagari qui fut l'un des aspects de l'opposition hindi/ourdou, pour n'évoquer que deux exemples. Les Xphonies constituent pour leur part des réalités politico-linguistiques incontestables qui peuvent peser sur l'ensemble de l'édifice gravitationnel. Face par exemple à la gestion linguistique des Nations Unies ou à celle de la Communauté européenne, la Francophonie a sa propre politique et nous allons voir que certaines alliances semblent se mettre en place.

\section{Geopolitique des langues romanes : etat des lieux ${ }^{7}$}

La notion de géopolitique des langues a été lancée par les géographes et géopoliticiens Yves Lacoste et Béatrice Giblin, en particulier dans un numéro de la revue Hérodote consacré essentiellement aux langues régionales et minoritaires et à leurs relations avec les langues nationales ${ }^{8}$. La même revue reviendra sur ce thème de façon plus large, à propos de la géopolitique de l'anglais en 2004 puis du français en $2007^{9}$. C'est pourquoi il n'est pas inutile de rappeler les positions d'Yves Lacoste, pour qui " la géographie ça sert d'abord à faire la guerre ", et qui a toujours insisté sur l'importance politique et stratégique de cette géographie, considérée comme instrument de pouvoir.

En ouverture du numéro d'Hérodote sur "Langues et territoires ", Béatrice Giblin écrivait :

"Si les sociolinguistes débattent des caractéristiques et évolutions lexicales et grammaticales de ces langues, ils s'intéressent aussi aux conditions de leur disparition ou de leur maintien, comme le font des historiens ou des anthropologues, les politologues et les juristes s'intéressant davantage à leur statut juridique. Mais si la langue est bien au centre de leurs préoccupations, le territoire où elle est censée être parlée est le plus souvent ignoré

7 Je reprends ici certains éléments d'un article que j'ai publié dans le numéro 75 de la revue Hermès, septembre 2016.

8 Hérodote $\mathrm{N}^{\circ} 105$, «Langues et territoires », 2002.

9 “Géopolitique de l'anglais”, Hérodote N¹15, 2005, “Géopolitique de la langue française”, Hérodote $\mathrm{N}^{\circ} 126,2007$. 
(sauf dans le cas de la Corse). Or, pour nous cet aspect de la question est essentiel. En effet, la question des langues régionales et minoritaires ne se limite ni à des querelles de linguistes, ni au nombre des locuteurs, ni à la défense d'un patrimoine culturel, même si tous ces aspects ont leur importance, elle est aussi affaire de territoire : sur quel territoire sont-elles parlées, ou le plus souvent, quel est le territoire sur lequel certains partisans de leur renaissance souhaiteraient les remettre en usage et pourquoi ? ${ }^{10}$

Et, présentant le numéro consacré à la "géopolitique de l'anglais », Y. Lacoste écrivait pour sa part :

"L'approche géopolitique d'une langue ne se borne pas à examiner sur la carte l'étendue de son extension et ses limites avec d'autres langues, à constater la coïncidence (ou la non coïncidence) avec les frontières de tel ou tel Etats. De surcroît, il est rare qu'une langue officielle soit la seule à être écrite et parlée par la population d'un Etat. En effet, dans chaque Etat, il y a aussi d'autres langues qui en fait sont parlées (et pas nécessairement écrites) par des groupes plus ou moins importants dont la localisation pose, elle aussi, nombre de problèmes"11.

En cela il ne se distinguait guère de la sociolinguistique mais, surtout, il focalisait sur une langue dominante (ici l'anglais) et sur ses rapports avec d'autres langues, dominées. Nous allons pour notre part évoquer non pas la géopolitique d'une langue mais celle d'un groupe de langues d'une même famille, d'un même groupe "génétique". Nous traiterons donc pour commencer de territoires, mais en modifiant légèrement la question de B. Giblin. Il ne s'agit pas en effet de savoir seulement sur quels territoires les langues sont parlées et quelles relations elles entretiennent avec d'autres langues du même territoire, mais aussi quelles relations ces langues dominantes entretiennent ou pourraient entretenir avec d'autres langues dominantes. Et la notion de langue n'étant jamais que la réification opérée par la linguistique de pratiques variées

10 op. cit. page 3

11 op. cit. page 5 
et parfois divergentes, c'est de groupe de locuteurs unis par le même type de pratiques linguistiques que nous traiterons, de leurs rapports de pouvoir sur un territoire et entre territoires.

La géographie des langues romanes est contrastée. Certaines d'entre elles, l'espagnol, le français et le portugais, sont implantées sur différents continents, tandis que d'autres, l'italien, le roumain, le catalan, le corse, les langues d'oc, le sarde, etc., ont une expansion très réduite, uniquement européenne et que peu d'entre elles sont internationales, au sens de "parlées dans plusieurs pays " (ce n'est le cas que du roumain en Roumanie et en Moldavie et de l'italien en Italie et dans une partie de la Suisse). Parler de géopolitique des langues romanes, c'est donc d'abord et essentiellement parler de trois grands ensembles, les ensembles francophone, hispanophone et lusophone, de trois langues qui disposent dans le monde de réseaux culturels (institut français, instituto camóes, instituto Cervantès), de chaînes de radio ou de télévision internationales (RFI, TV5, France 24 pour le français, Antenna 3, TVE internacional pour l'espagnol, RTP internacional, Globo internacional pour le portugais etc.) et d'organisations politiques.

Voici une carte montrant l'expansion de ces trois langues à partir d'une petite portion de l'Europe sud-occidentale vers l'Afrique et l'Amérique, présentant les pays dans lesquels elles sont officielles ou co-officielles.

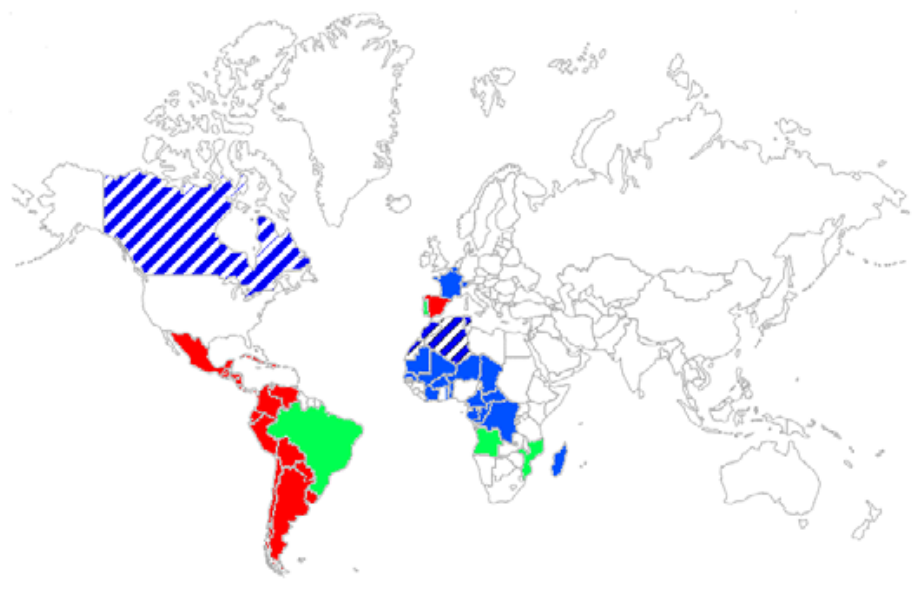

1. Carte de l'expansion de l'espagnol, du français et du portugais. 
Ces trois grands espaces linguistiques ont un certain nombre de caractéristiques communes. Ils sont le produit d'un passé colonial ou impérial, ils s'étendent sur trois ou quatre continents, ils sont plus ou moins organisés politiquement et ils produisent de la diversité, chacune des langues prenant des formes locales dont certaines, comme le portugais au Brésil, on beaucoup plus de locuteurs que la forme d'origine. Mais ils different sur un point majeur : si l'Espagne est minoritaire dans l'ensemble des hispanophones natifs, si le Portugal l'est encore plus dans l'ensemble des lusophones, la France reste majoritaire dans l'ensemble des francophones première langue. Et, dans chacun de ces trois espaces, les trois langues qui les constituent prennent des formes locales, pour une raison évidente : plus un " empire " a une origine ancienne et plus sa langue s'y est implantée, passant lentement du statut de langue exogène à celui de langue endogène par un processus que j'ai baptisé acclimatation ${ }^{12}$.

Il en résulte un positionnement différencié du lieu de pouvoir normatif. Le Brésil, par son poids démographique, est en situation d'imposer sa norme à l'ensemble des lusophones, avec des retombées économiques non négligeables, par exemple dans le domaine de l'édition. Ainsi dans l' "accord orthographique de la langue portugaise ", adopté entre 2004 (par le Brésil) et 2008 (par le Portugal), qui concerne environ 2.000 mots et tend à rapprocher l'orthographe de la prononciation en supprimant des consonnes non prononcées, sur le modèle déjà appliqué au Brésil, 75\% des changements portent sur des formes utilisées au Portugal. La norme de l'espagnol fluctue pour sa part d'un pays à l'autre, on ne double pas les films de la même façon pour l'Espagne et pour l'Amérique latine, des ouvrages sont traduits différemment en Espagne et dans certains pays latino-américains, etc. Quant à la norme du français, elle est plutôt le fait de la France ou, plus largement, des pays francophones du Nord.

Toutes les conditions sont donc réunies pour qu'il y ait une guerre des normes, ou des standards, entre l'Europe et l'Amérique pour l'espagnol et le portugais. Ainsi l'Académie Royale espagnole se préoccupe d'une part de défendre la norme ibérique (elle a par exemple publié en 2005 un Diccionario panhispanica de dudas) mais elle a d'autre part créé des « académies correspondantes » dans tous les autres pays hispanophones (y compris aux Etats-

12 Voir L-J Calvet, Pour une écologie des langues du monde, Paris Plon 1999 pp 142-43 
Unis) qui pourraient défendre des normes locales. En revanche le problème ne se pose guère entre le Nord et le Sud de la francophonie, et il n'existe pas d'académie de la langue française du Congo, du Mali ou du Sénégal. Lorsque des mots ou des expressions venues de pays africains francophones entrent dans un dictionnaire français, c'est par décision d'éditeurs ou de lexicologues français et non pas africains : le Nord, et en particulier la France, garde le pouvoir sur la norme du français.

\section{Jeux de cooperation et jeux de lutte}

Si nous classons les langues du monde selon le nombre de pays dans lesquels elles sont officielles l'anglais vient à la première place, immédiatement suivi par le français, l'espagnol et le portugais. Le français est la langue officielle, co-officielle ou nationale de 31 pays ou régions. L'espagnol pour sa part l'est dans 22 Pays et le portugais dans 9. Les trois langues jouent donc un rôle officiel dans 62 pays, et si nous y ajoutons l'italien (Italie, SaintMarin, Suisse, Vatican, Slovénie, Croatie), le roumain (Roumanie, Moldavie, Serbie) et le catalan (Espagne, Andorre), nous arrivons à plus de 70 territoires. Tout cela constitue à l'échelon international un groupe de pression potentiel non négligeable. L'ensemble des pays ayant une langue romane pour langue officielle représente par exemple plus d'un tiers des pays membres de l'ONU ou de l'UNESCO, ce qui leur donnerait s'ils se concertaient pour défendre des positions communes sur des thèmes à élaborer une force politique certaine.

On distingue dans la théorie des jeux entre des jeux de coopération, dans lesquels les joueurs ont des intérêts convergents, et des jeux de lutte, dans lesquels les joueurs ont des intérêts divergents. Dans le premier cas il s'agit de jeux à somme positive, chacun gagnant, mais bien sûr ne gagnant pas la même chose, et dans le second cas de jeux à somme nulle, les uns gagnant ce que les autres perdent. Que pourrait nous apporter la transposition de cette distinction dans le domaine des langues?

On perçoit aisément les retombées économiques ou militaires de la constitution (en général coloniale) de ces espaces. Du côté économique on peut songer à l'implantation de l'entreprise de télécommunication espagnole Telefonica en Amérique latine, à la création en Amérique latine d'un marché commun du Sud, le MERCOSUR, au franc CFA, zone franc d'Afrique centrale 
(CEMAC) et d'Afrique de l'Ouest (UEMOA) ou encore à l'implantation de l'entreprise pétrolifère française Total en Afrique francophone (Côte d'Ivoire, Gabon, Congo). On peut aussi songer à la possibilité, vue du Sud, de jouer la concurrence économique entre différents acteurs du Nord, par exemple la France et le Canada francophone. Et du point de vue militaire on peut évoquer les interventions françaises au Mali ou en Centrafrique. Mais tout ceci n'a pas de rapports directs avec les langues, tout au plus peut-on constater que quand la colonisation n'a pas laissé beaucoup de traces linguistiques (Vietnam ou Cambodge pour la France, Macao ou Timor oriental pour le Portugal) les relations politiques ou économiques sont plus distendues. Nous sommes en fait confrontés ici aux versants économique ou militaire d'une expansion dont nos " trois espaces" sont le versant linguistique.

Conçue en termes de jeu à somme nulle, la géopolitique des langues mènerait soit, dans un axe horizontal, à une lutte entre trois langues dominantes, chacune d'entre elles cherchant à occuper la seconde place derrière l'anglais, soit, dans un axe vertical, à une lutte entre une langue dominante et les langues qu'elle domine (par exemple le français face aux langues africaines, l'espagnol face aux langues indiennes, le portugais faces aux langues indiennes et aux langues africaines).

Conçue en termes de jeu à somme positive, elle pourrait mener dans l'axe horizontal à la recherche d'une défense commune des trois langues dominantes (avec le danger d'un Yalta linguistique, une sorte de partage du monde face à l'appétit anglophone) et/ou dans l'axe vertical à la recherche d'une coexistence entre langues partenaires qui soit au bénéfice de chacune d'entre elles. La question est bien sûr de savoir si, la géographie servant d'abord à faire la guerre, comme l'a écrit Yves Lacoste, elle sert également à faire la "guerre des langues » ou si, revisitée par la politique linguistiques, elle peut mener vers une " paix des langues ». Cette question est aujourd'hui ouverte, dans un processus dont nous sommes ou pouvons être les acteurs.

Recebido em: 05/11/2016 Aprovado em: 09/12/2016 